\title{
Variables clínicas asociadas al diagnóstico de meningo-encefalitis herpética en pacientes adultos: un estudio anidado de casos y controles
}

\author{
María de las Mercedes Magaz, Vanina Wainsztein, Joaquín Maritano, María Nelly Gutiérrez, \\ Fernando Binder, Bruno L. Ferreyro, Federico Angriman y Gabriel Waisman
}

\begin{abstract}
Hospital Italiano de Bueno Aires. Buenos Aires, Argentina. Departamento de Medicina Interna (MdelasMM, VW; JM, MNG, F,B $B L F, F A, G W)$.

Universidad de Buenos Aires,

Buenos Aires, Argentina. Cátedra de Farmacología (BLF, FA)

Recibido: 20 de agosto de 2014 Aceptado: 13 de marzo de 2015

Los autores declaran no poseer conflictos de interés.
\end{abstract}

Establecimiento donde se realizó e estudio: Hospital Italiano de Buenos Aires. Buenos Aires, Argentina.

Correspondencia a: María de las Mercedes Magaz mercedes.magaz@hospitalitaliano.

\section{Clinical characteristics of adult patients with herpetic meningoencephalitis: a nested case control study}

Introduction: Herpes simplex virus (HSV) is the most common etiology of sporadic encephalitis and presents with an estimated mortality of 50-70\%. Objective: To describe baseline characteristics of patients with herpetic encephalitis admitted to a tertiary teaching hospital and their difference with patients with non herpetic encephalitis. Materials and Methods: Nested case control study using a retrospective cohort of patients with suspected encephalitis admitted to the Hospital Italiano de Buenos Aires (2006-2013). Adult patients included had a lumbar puncture with a positive or negative polimerase reaction for HSV. A case of herpetic encephalitis was defined as a positive polimerase reaction in spinal fluid. For each case, 5 controls were randomly selected. Results: There were no baseline differences present between cases and controls. The only covariate associated with herpetic encephalitis was an abnormal brain magnetic resonance imaging (MRI) (OR: 5.37, IC 95\% 1.42-20.38, p < 0.01). The most frecuent alterations in the MRI were extratemporal lesions or temporal ones with or without haemorrhage. Discussion: There are no apparent baseline clinical differences between patients with or without herpetic encephalitis. A positive finding in a brain MRI should be taken into account during clinical workup.

Key words: Herpes simplex virus, meningoencephalitis, herpetic, encephalitis, prognosis.

Palabras clave: Virus herpes simplex, encefalitis herpética, factores pronósticos.

\section{Introducción}

$\mathrm{E}$ 1 virus herpes simplex (VHS) es la causa reportada más común de encefalitis esporádica en los Estados Unidos de América ${ }^{1,2}$. La incidencia acumulada de encefalitis herpética $(\mathrm{EH})$ es aproximadamente 0,2 cada 100.000 personas/año y presenta una mortalidad entre 50 y $70 \%{ }^{1,3,4}$. Con terapia empírica temprana (definida como la terapéutica previa a la pérdida de conciencia del paciente, dentro de las $24 \mathrm{~h}$ del inicio del cuadro o cuando el paciente presenta una escala de coma de Glasgow entre 9 y 15) la misma se reduce a $20-30 \% 0^{3,5-7}$. Asimismo, varios trabajos prospectivos han observado que la terapia empírica temprana mejora el estatus funcional del paciente al alta. Esto demuestra la relevancia que tiene el diagnóstico precoz en estos pacientes y el adecuado reconocimiento de los signos, síntomas, alteraciones del laboratorio, en líquido cefalorraquídeo (LCR) o en las imágenes del sistema nervioso central (SNC) realizadas ${ }^{2,3,6,7}$.

La reacción de polimerasa en cadena (RPC) para VHS en el LCR es el método de elección para realizar el diagnóstico de EH con una sensibilidad y especificidad mayores a $95 \% 0^{3,4}$. Se han postulado diversas variables clínicas predictoras del diagnóstico de EH pero con gran diversidad en su reporte ${ }^{7,8}$. Más aún, algunos estudios han reportado variables predictoras de peor pronóstico tales como la escala "Acute Physiology Score" $>27$ al momento de la consulta y la demora de más de $48 \mathrm{~h}$ desde el inicio del cuadro hasta el inicio de la terapia. Otros estudios demuestran que más allá de estos predictores clínicos, cerca de $40 \%$ de los sobrevivientes queda con secuelas tales como alteraciones en la conducta, la memoria y el aprendizaje, convulsiones, anormalidades neuropsicológicas, disfunción motora fina y eventos trombo-embólicos ${ }^{9}$. El tratamiento de soporte es crucial así como tener un protocolo que identifique pacientes en riesgo de tener $\mathrm{EH}$. No conocemos adecuadamente la epidemiología ni las características de la enfermedad en nuestro medio. Esto resultaría fundamental para poder modificar la evolución de la enfermedad y sus consecuencias posteriores.

El objetivo principal de nuestro estudio fue describir las características de nuestra población con diagnóstico final de EH. Asimismo, detectar variables clínicas basales asociadas a su diagnóstico y evaluar la terapéutica instaurada, la evolución de los pacientes y su potencial relación con la gravedad de las secuelas neurológicas. 
Nuestro objetivo final es poder contribuir al diagnóstico precoz y la administración temprana de tratamiento específico.

\section{Materiales y Métodos}

Diseño. Se utilizó un diseño anidado de casos y controles sobre una cohorte retrospectiva de pacientes adultos admitidos a un centro universitario de tercer nivel: el Hospital Italiano de Buenos Aires, Argentina. Dicha cohorte se encuentra conformada específicamente por todos los pacientes que acudieron a la central de emergencias de adultos del Hospital Italiano de Buenos Aires entre los años 2006 y 2013, con diagnóstico presuntivo de encefalitis, a quienes se les realizó una punción lumbar y se solicitó una RPC para VHS en el LCR. Por cada caso (RPC positiva para VHS) se tomaron cinco controles en forma aleatoria de la cohorte inicial. El presente estudio fue aprobado por el Comité local de Ética de Protocolos de Investigación (CEPI, número de protocolo: 2224) y fue realizado de acuerdo con la declaración de Helsinki y reportado según las guías $\mathrm{STROBE}^{10}$.

Población. Se incluyó en el estudio a todos los pacientes sobre 17 años de edad que fueron admitidos en la central de emergencias de adultos entre los años 2006 y 2013 a los cuales se les hubiera realizado una punción lumbar y en la misma se hubiera solicitado RPC para VHS.

Dicha punción lumbar se realizó en respuesta a la presunción clínica de encefalitis, basada en la presencia de uno o más de los siguientes signos o síntomas: fiebre, cefalea, deterioro del sensorio, convulsiones y la presencia de hallago imagenológico característicos en las imágenes de cerebro realizadas. Se definió como caso confirmado a los pacientes que presentaron RPC positiva para VHS en el LCR.

Mediciones y variables explanatorias. Los datos demográficos y las características clínicas de los pacientes se obtuvieron de una base de datos secundaria electrónica. Se registraron de cada paciente variables demográficas, clínicas, de laboratorio y de imágenes en una base de datos electrónica. La revisión de historias clínicas fue realizada por dos médicos residentes y cuatro médicos del Servicio de Clínica Médica del Hospital Italiano de Buenos Aires. El ingreso de los datos se realizó por dos operadores independientes con posterior conciliación de los datos por el investigador principal, a fin de detectar valores erróneos e información faltante. Específicamente, se registraron: edad, sexo, co-morbilidades basales pre-existentes, escala de Katz (valoración funcional del anciano de 0 a 6), características clínicas al inicio del cuadro y variables de laboratorio como función renal y hepatocelular. Asimismo, se registró el resultado del análisis físico-químico, citológico y de la RPC para VHS en el LCR. A su vez, se registraron características evolutivas de ambas poblaciones y la administración temprana $(<48 \mathrm{~h})$ o no de tratamiento con aciclovir. Por último, se registró el resultado de estudios complementarios realizados: tomografía axial computada (TAC) y/o resonancia magnética (RM) de cerebro.

A priori, se consideraron potencialmente asociadas al diagnóstico de $\mathrm{EH}$ a las siguientes co-variables: fiebre, cefalea, inmunosupresión, edad avanzada y la presencia de hallazgos imagenológicos característicos en las imágenes de cerebro realizadas.

Evento primario: Se definió como evento primario (caso) a la ocurrencia de la RPC positiva para VHS (tipo 1 ó 2) en el LCR luego de la punción lumbar. Por cada caso registrado se incluyeron cinco controles aleatoriamente seleccionados.

Análisis estadístico: Las variables cuantitativas continuas se resumieron utilizando media y desvío estándar o mediana e intervalo intercuartil según la distribución observada, mientras que las variables categóricas se resumieron utilizando proporciones. El análisis bivariado para evaluar la asociación de las principales co-variables con la ocurrencia de EH se llevó a cabo utilizando test de student o Wilcoxon para variables continuas según la distribución observada y el test exacto de Fisher para las variables categóricas. Para identificar variables asociadas a la presencia de EH se estimaron odds ratio con sus respectivos intervalos de confianza para cada variable. Por último, se realizó un análisis multivariado utilizando un modelo de regresión logística donde se incluyeron variables clínicas consideradas a priori asociadas al evento, así como también aquellas variables asociadas al mismo en el análisis bivariado. El tamaño de muestra fijo fue de 17 casos en el periodo estudiado. Se seleccionaron cinco controles por cada caso incluido. Se tomó como nivel de significancia estadística a una $\mathrm{p}<0,05$.

\section{Resultados}

En el período de tiempo estudiado (2006-2013) se realizó una punción lumbar con solicitud de RPC para VHS a 753 pacientes que acudieron a la central de emergencias del Hospital Italiano de Buenos Aires. El número de pacientes adultos con RPC positiva (EH) fue de 17. El número de pacientes sobre el cual se realizó el análisis fue de 116 (Figura 1).

Con respecto a las características basales de los pacientes con diagnóstico de EH (Tabla 1), 41,2\% de los pacientes fueron hombres y la media de edad fue de 56,8 años. Todos los pacientes presentaron una escala de actividades de la vida diaria de $6 / 6$. Solamente $11,8 \%$ de los pacientes tenían diagnóstico de demencia o deterioro 
Figura 1. Flujo de pacientes. Abreviaturas: VHS: virus herpes simplex, PL: punción lumbar. RPC: reacción de polimerasa en cadena.

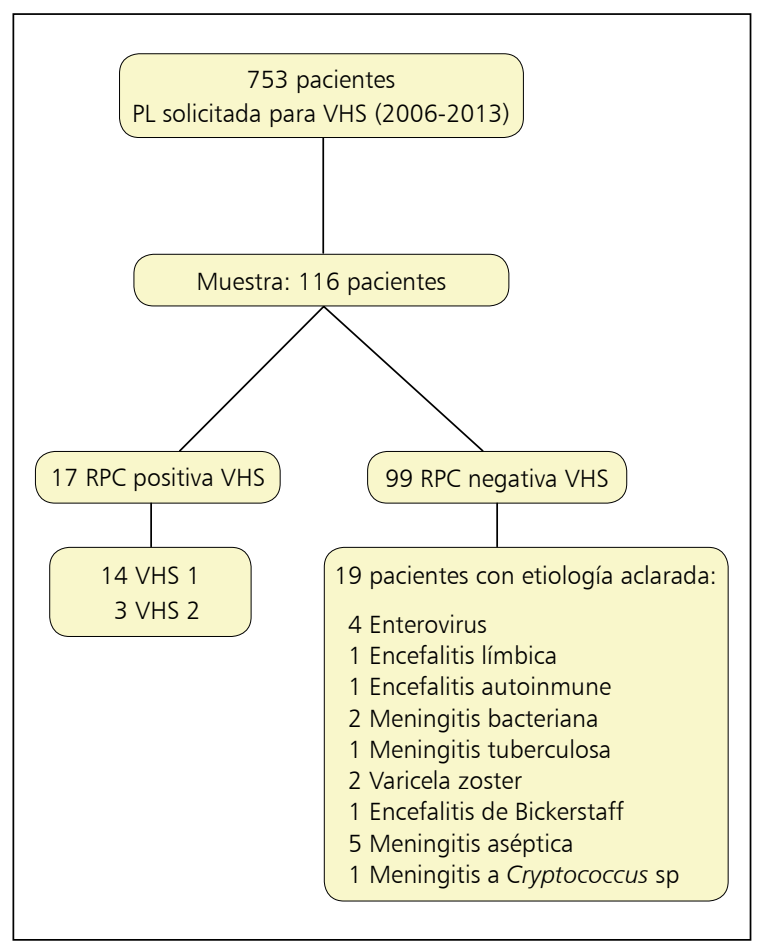

cognitivo. Las co-morbilidades previas al momento de la punción lumbar más frecuentes fueron: inmunosupresión $(35,3 \%)$ y enfermedad oncológica $(23,5 \%)$. Los síntomas iniciales que predominaron en nuestra población fueron: confusión $(52,9 \%)$, fiebre $(52,9 \%)$, cefalea $(47,1 \%)$ y deterioro del sensorio $(29,4 \%)$. La media de celularidad en el LCR hallada fue de 167,5 céls $/ \mathrm{mm}^{3}$ (RIC 1-300), con un valor medio de glucorraquia de $65,8 \mathrm{mg} / \mathrm{dl}(\mathrm{DE} 33,0)$ y $82 \mathrm{mg} / \mathrm{dl}$ (RIC: $54-124,5$ ) de proteinorraquia. El 53,8\% de los pacientes tuvo lesiones en la RM de encéfalo, siendo hipodensidades temporales o hemorrágicas en $38,5 \%$ y extra-temporales en $15,4 \%$. El resto de los pacientes a los que se les realizó RM tuvo otros hallazgos no vinculables al diagnóstico de $\mathrm{EH}$.

Con respecto al tratamiento, $76,4 \%$ de los pacientes con EH fueron tratados con aciclovir, en casi $60 \%$ de los casos fue iniciado de manera precoz. El 30\% de los pacientes de nuestra población fueron atendidos en unidades de cuidados críticos. Con respecto a su evolución, menos de $15 \%$ de los pacientes presentó alguna secuela neurológica y ningún paciente murió. Solamente tres casos fueron causados por VHS 2.

Se detectaron diferencias mínimas y estadísticamente no significativas en las características demográficas y en las co-morbilidades de los pacientes con EH comparados con aquellos con RPC negativa. Dentro de los síntomas iniciales, la cefalea estuvo presente en $47,1 \%$ de los pacientes con EH contra 22,2\% en los pacientes con RPC negativa (p: 0,04). En el LCR se observaron también
Tabla 1. Características basales de los pacientes con encefalitis herpética

Co-variables
Características demográficas
Edad, años (media, DE)
Género masculino ( $\mathrm{n}, \%)$
Co-morbilidad previa
Lugar de residencia-Institucionalización ( $\mathrm{n}, \%)$
Actividades de la vida diaria (mediana, RIC)
Deterioro cognitivo ( $\mathrm{n}, \%)$
Enfermedad oncológica ( $\mathrm{n}, \%)$
Inmunosupresión ( $\mathrm{n}, \%)$
Insuficiencia renal crónica (n, \%)

Síntomas iniciales (n, \%)

Fiebre

Cefalea

Foco neurológico

Confusión

Convulsiones

Coma

VHS positivo

n: 17

\section{Estudios de laboratorio}

Glicemia, mg/dl (media, DE)

$0 \quad(0)$

\section{Líquido cefalorraquídeo}

Glucorraquia, mg/dl (media, DE)

Proteinorraquia, g/dl (mediana, RIC)

Celularidad, células $/ \mathrm{mm}^{3}$ (mediana, RIC)

\section{Otros métodos complementarios}

\section{Alteraciones en la RM ( $n, \%)$}

Lesión temporal o hemorragia

Lesión extratemporal

Alteraciones en el ECG $(n, \%)$ *

Tratamiento y evolución clínica ( $n, \%)$

Tratamiento con aciclovir

Tratamiento precoz

Muerte durante el seguimiento

Abreviaturas: DE: desvío estándar, RIC: intervalo intercuartil (percentilo 25-75). ECG: electrocardiograma. RM: resonancia magnética, RPC: reacción en cadena de polimerasa, VHS: herpes virus. *Número total de pacientes con RPC y EEG realizado: 4.

diferencias no significativas en la proteinorraquia y la celularidad. El 53,8\% de los pacientes con EH presentó alteraciones en la RM de cerebro vs $18,3 \%$ en pacientes con RPC negativa en LCR (p: 0,01$)$. En los pacientes con $\mathrm{EH}$, el patrón más observado fueron las lesiones extratemporales o lesiones temporales, con o sin hemorragia. De los pacientes con EH, aproximadamente $80 \%$ recibió tratamiento con aciclovir y en más de la mitad de los mismos, de forma precoz.

Finalmente, en el modelo multivariado de regresión logística (Tabla 3) la única variable asociada a la presencia de un diagnóstico de EH fue la presencia de alteraciones sugerentes en la RM de cerebro (OR: 5,37, IC 95\% 1,42$20,38, p<0,01)$. 


\section{Tabla 2. Odds ratio (OR) e intervalo de confianza (IC) del 95\% para presentar encefalitis herpética}

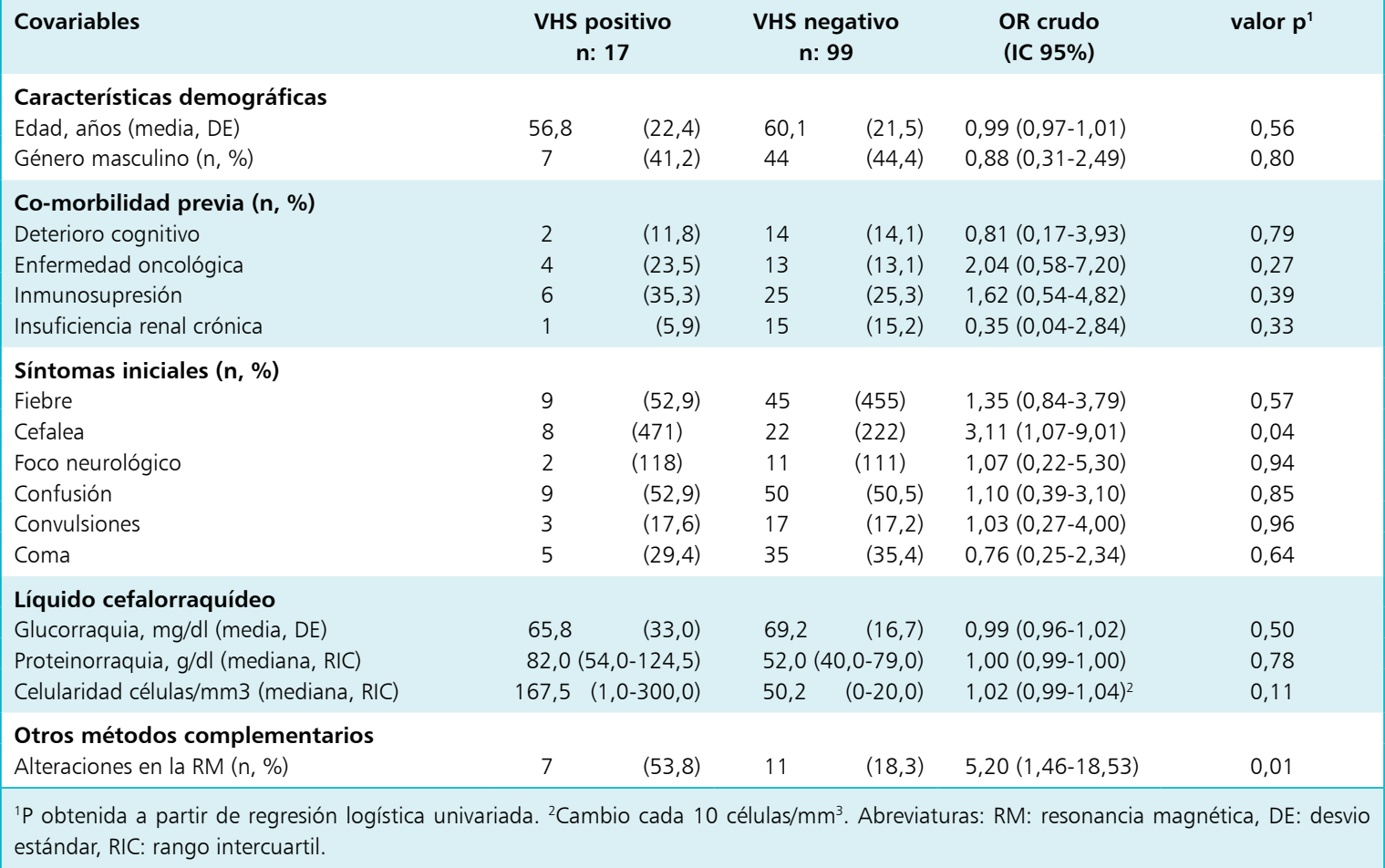

\section{Discusión}

Este estudio nos permitió conocer mejor la epidemiología y las características clínicas de la EH en nuestro medio comparando nuestra población con diagnóstico definitivo de EH y evaluando sus diferencias respecto de aquellos pacientes en los que existió la sospecha diagnóstica y resultó no ser EH el diagnóstico final. A su vez, permitirá realizar una primera aproximación acerca de diferentes co-variables de posible utilidad para el diagnóstico de la enfermedad.

La población del estudio tenía una edad media de aproximadamente 60 años, sin demasiada carga de co-morbilidad y no hubo caso fatal alguno dentro de los pacientes con diagnóstico de EH. Esto, si bien no coincide con la literatura científica existente ${ }^{3,7,11}$, nos muestra las características de nuestra población enferma. Existen varias posibles explicaciones para esta dicotomía, incluyendo en primer lugar, el posible sesgo de selección inicial; nuestro hospital es un centro de derivación y los pacientes incluidos eran relativamente jóvenes y con poca carga de co-morbilidad. En segundo lugar, el sesgo de información correspondiente a los pacientes que ingresaron con síntomas de gravedad a la central de emergencias y a los cuales no se les realizó test diagnóstico por limitación
Tabla 3. Odds ratio (OR) e intervalo de confianza (IC) del $95 \%$ ajustado para presentar encefalitis herpética

\begin{tabular}{|lcc|}
\hline Covariable candidata & OR ajustado (IC 95\%) & valor $\mathbf{p}^{\mathbf{1}}$ \\
\hline Edad & $0,99(0,96-1,02)$ & 0,74 \\
Lesiones en RM & $5,37(1,42-20,38)$ & 0,01 \\
Cefalea & $3,76(0,92-15,32)$ & 0,08
\end{tabular}

'Modelo multivariable basado en regresión logística incluyendo las siguientes covariables: edad, cefalea, lesiones en MRI.

terapéutica o la gravedad en su presentación clínica. En tercer lugar, cabe la posibilidad de mejor manejo, adecuado y temprano que impacte en el seguimiento y pronóstico de los pacientes. Esto seguramente genera que se excluyan con mayor frecuencia aquellos casos más graves, con mayores secuelas o que hayan tenido un desenlace fatal en su evolución.

Con respecto a las variables asociadas al diagnóstico de EH, tanto la cefalea como las alteraciones en el LCR fueron encontradas más frecuentemente en los pacientes con $\mathrm{EH}$ pero esta diferencia no se mantuvo significativa en el análisis multivariado. En cuanto a las imágenes del 
SNC, la RM fue la única de todas las variables analizadas en este estudio que arrojó un resultado estadísticamente significativo en su comparación con la población sin EH. Podríamos concluir, basándonos en estos resultados, que durante la evaluación de un paciente con sospecha de EH, si bien una RM de encéfalo normal no descarta el diagnóstico, una RM patológica lo vuelve potencialmente más probable. El costo, la duración y la baja disponibilidad de la misma serían serios limitantes para su uso extendido; no obstante, podría ser utilizada como estudio de elección para confirmación diagnóstica en aquellos casos con alta sospecha clínica y RPC en LCR negativa para EH o en aquellos casos dudosos por clínica y/o resultado de LCR.

Finalmente, con respecto al tratamiento, en nuestra cohorte la mayoría de los pacientes con diagnóstico de EH recibió tratamiento con aciclovir y de manera precoz. Estos datos se asemejan a los encontrados en la literatura médica $^{3,711}$ y pueden explicar, en parte, la evolución satisfactoria de nuestros pacientes.

El estudio presenta varias limitaciones potenciales. En primer lugar, la posibilidad de sesgos de selección inicial que dificulten la comparación de ambos grupos es inherente al diseño retrospectivo de casos y controles, más aún utilizando una base de datos secundaria. Esto dificulta el arribo de conclusiones con impacto en la clínica. Sin embargo, destacamos la utilidad del análisis de variables clínicas, de laboratorio, de LCR y de imágenes respecto de la población con diagnóstico de EH y su comparación con aquellos pacientes con características similares en los cuales se descartó este diagnóstico, como generador de hipótesis y nuevos estudios prospectivos. En segundo lugar, la incapacidad de medir variables clínicas y de laboratorio ausentes en el registro o la historia clínica electrónica impide la inclusión de dicha información en el modelo. En tercer lugar, debido a la característica retrospectiva de nuestro estudio, cabe la posibilidad de que pacientes con alta sospecha clínica de $\mathrm{EH}$ con resultado negativo en la RPC en LCR hayan sido clasificados como controles cuando en realidad representaban casos. Esto incluye un posible sesgo de mis-clasificación y puede subestimar las diferencias encontradas entre casos y controles de EH. Dicha mis-clasificación podría resolverse en la práctica diaria utilizando el control de LCR a las $48 \mathrm{~h}$ en aquellos pacientes con alta sospecha clínica y resultado negativo inicial, así como también la determinación de anticuerpos específicos en LCR. En cuarto lugar, hemos incluido pacientes de un solo centro lo que teóricamente disminuye la validez externa haciendo a nuestra población no necesariamente generalizable a otras. Por último, al tener un número reducido de eventos, este estudio carece potencialmente de poder para observar diferencias entre las poblaciones que quizás con una muestra más grande se harían evidentes.
En contrapartida, nuestro estudio presenta varias fortalezas. En primera instancia, es el primer trabajo que evalúa las características basales de los pacientes con $\mathrm{EH}$ en nuestro medio lo que resulta de importancia epidemiológica, tanto para el diagnóstico precoz como para la asignación de probabilidades previas de anclaje durante dicho proceso. En segunda instancia, el listado de variables potencialmente asociadas al diagnóstico de EH actúa como disparador de hipótesis que sirvan para ayudar al diagnóstico correcto y temprano de esta entidad. Por último, la inclusión de controles aleatorizados de una gran cohorte retrospectiva a lo largo de varios años mejora la comparabilidad entre los dos grupos y reduce sustancialmente el sesgo de selección inicial inherente al proceso.

En conclusión, nuestro estudio compara las características clínicas de los pacientes con y sin EH no detectando diferencias importantes. Los resultados sugieren que la realización de una RM de encéfalo en la etapa diagnóstica de EH tiene potencial aplicación en la práctica clínica, si bien la espera de la misma no debería retrasar el comienzo de la terapéutica precoz adecuada. La misma puede tener un valor de importancia para confirmar el diagnóstico en casos donde existe la duda de $\mathrm{EH}$ a pesar de otros tests diagnósticos ya realizados.

\section{Resumen}

Introducción: El virus herpes simplex (VHS) es la causa reportada más común de encefalitis esporádica con una mortalidad estimada de 50 a 70\%. Objetivo: Describir las características de los pacientes con encefalitis herpética (EH) en nuestro medio y sus variables clínicas asociadas. Materiales y Métodos: Estudio anidado de casos y controles sobre una cohorte retrospectiva en el Hospital Italiano de Buenos Aires (2006-2013). Se incluyeron pacientes adultos con sospecha de encefalitis a quienes se les realizó una punción lumbar con posterior reacción de polimerasa en cadena para VHS en líquido cefalorraquídeo. Por cada caso (reacción positiva para VHS) se tomaron cinco controles aleatoriamente seleccionados. Resultados: No se observaron diferencias significativas en las características demográficas y clínicas entre los casos de $\mathrm{EH}$ y los controles. La única variable clínica asociada al diagnóstico de $\mathrm{EH}$ fue la alteración en la resonancia magnética (RM) de cerebro (OR: 5,37, IC 95\% 1,42$20,38 ; p<0,01)$. Los patrones más comunes de alteración en la RM fueron las lesiones extra-temporales o lesiones temporales con o sin hemorragia. Discusión: Este hallazgo nos hace jerarquizar el hallazgo de un resultado positivo en la RM durante la valoración inicial de un paciente con clínica compatible de $\mathrm{EH}$. 


\section{Referencias bibliográficas}

1.- Sili U, Kaya A, Mert A, Group HSVES Herpes simplex virus encephalitis: clinical manifestations, diagnosis and outcome in 106 adult patients. J Clin Virol 2014; 60 (2): 112-8.

2.- Bloch K C, Glaser C. Diagnostic approaches for patients with suspected encephalitis. Curr Infect Dis Rep 2007; 9 (4): 315-22.

3.- Kennedy P G, Steiner I. Recent issues in herpes simplex encephalitis. J Neurovirol 2013; 19 (4): 346-50.

4.- $\quad$ Tyler K L. Update on herpes simplex encephalitis. Rev Neurol Dis 2004; 1 (4): 169-78.
5.- Rozenberg F. Acute viral encephalitis. Handbook of Clinical Neurology 2013; 112: 1171-81.

6.- Tunkel A R, Glaser C A, Bloch K C, Sejvar J J, Marra C M, Roos K L, et al. The management of encephalitis: clinical practice guidelines by the Infectious Diseases Society of America. Clin Infect Dis 2008; 47 (3): 303-27.

7.- Sheybani F, Arabikhan H R, Naderi H R. Herpes simplex encephalitis (HSE) and its outcome in the patients who were admitted to a tertiary care hospital in Mashhad, Iran, over a 10-year period. J Clin Diagn Resh: JCDR 2013; 7 (8): 1626-8.

8.- Cunha B A. The clinical and laboratory diagnosis of acute meningitis and acute encephalitis. Expert Opin Med Diagn 2013; 7 (4): 343-64.

9.- Terlizzi V, Improta F, Di Fraia T, Sanguigno E, D'Amico A, Buono S, et al. Primary herpes virus infection and ischemic stroke in childhood: A new association? J Clin Neurosci. J Neurosurg Soc Austr 2014.

10.- Vandenbroucke J P, Von Elm E, Altman D G, Gotzsche P C, Mulrow C D, Pocock S J, et al. [Strengthening the reporting of observational studies in epidemiology (STROBE): explanation and elaboration]. Gaceta sanitaria/SESPAS 2009; 23 (2): 158.

11.- Steiner I, Benninger F. Update on herpes virus infections of the nervous system. Curr Neurol Neurosci Rep 2013; 13 (12): 414. 